\title{
Analysis of reduction status and lag screw direction in intertrochanteric fractures treated with intramedullary nailing: Possible influence of anterior femoral head-neck offset
}

\author{
Ikufumi Yamada $^{1 *}$, Takehiro Matsubara ${ }^{2}$ and Sakae Tanaka ${ }^{2}$ \\ ${ }^{1}$ Department of Orthopedic Surgery, Fushimi Momoyama General Hospital, Kyoto, Japan \\ ${ }^{2}$ Department of Orthopedic Surgery, The University of Tokyo, Tokyo, Japan
}

\begin{abstract}
Aim: There are many evaluations of lag screw tip position. However, there is no comprehensive report on the evaluation of the alignment of the lag screw position relative to the cervical axis from the nail hole to the femoral head. Purpose of this study is to analyze the relationship between the reduction status and the alignment of the lag screw in patients with intertrochanteric fractures treated with intramedullary nails (IHN), using postoperative computed tomography (CT).

Patients and methods: Postoperative CT evaluation was performed in 100 intertrochanteric fractures (23 men and 77 women), which were randomly selected from 237 cases, treated with IHN. The mean patients' age at the time of surgery was mean age $83.9 \pm 8.9$ years (61 to 96). The position of the nail, fracture reduction status, and direction of the screw relative to the femoral neck were assessed by axial plane CT. The angle between the screw and the femoral neck axis (deviation angle) was measured and deemed positive with the presence of anterior deviation.

Results: The proximal fragment was posteriorly displaced in 82 patients, anteriorly displaced in one patient, and nondisplaced in 17 patients. The lag screw was positioned at the anterior third relative to the femoral neck in eight patients, the middle third in 15 patients, and the posterior third in 77 patients. The lag screw was deviated in 61 patients, with a mean deviation angle of $11.6 \pm 10.9^{\circ}$ (7.2 to 37.5$)$. When the screw was inserted parallel with the femoral neck, $84.6 \%$ (34/39) of the patients had displaced fragments, and most (33 patients) were posteriorly displaced. In 17 nondisplaced cases, posterior to anterior deviation of the lag screw direction was observed in 12 patients $(70.6 \%)$.

Discussion: Anatomical reduction and proper positioning of the lag screw were rarely achieved simultaneously in IHN surgeries, possibly because of anterior offset of the femoral neck relative to the femoral shaft. New concepts in intramedullary nail design such as nails with an anterior offset will be required to achieve better CT results with IHN.
\end{abstract}

\section{Introduction}

A dramatic increase in the number of osteoporotic fractures has been recognized in aging populations [1-3]. Among osteoporotic fractures, hip fractures are a leading cause of disability, morbidity, and mortality in older patients, and are now a global burden in public health $[4,5]$. Intramedullary hip nailing (IHN) is popular for internal fixation of intertrochanteric fractures because of its advantages, namely, efficient load transfer, less soft tissue damage, and shorter operative time compared with sliding hip screws, and is preferentially used for reverse oblique intertrochanteric fractures [6-9]. However, previous studies reported that IHN causes complications such as loss of reduction and cut-out, and that IHN is associated with a higher risk of intraoperative and later femoral fractures and higher reoperation rates compared with sliding hip screws $[10,11]$. In addition, several studies reported that rotational malalignment was frequently observed after IHN surgeries [12-14]. Unstable fracture type, non-anatomical reduction, nonoptimal lag screw positioning, and tip-apex distance $>25 \mathrm{~mm}$ were identified as risk factors for cut-out after IHN $[15,16]$.
Although the optimal surgical technique for IHN is still controversial, researchers agree that anatomical reduction of the fracture and proper positioning of the lag screw are critical for successful treatment, and that the center of the femoral neck on the lateral view is considered the ideal position for the lag screw [17-20]. There are many evaluations of screw tip position of the lag screw [1720]. However, there is no comprehensive report on the evaluation of the alignment of the lag screw relative to the cervical axis from the nail hole to the femoral head. The objective of the present study was to evaluate reduction status and the positioning of the lag screw in patients with intertrochanteric fractures treated using IHN and evaluated with postoperative computed tomography (CT).

${ }^{*}$ Correspondence to: Ikufumi Yamada, Department of Orthopedic Surgery, Fushimi Momoyama General Hospital, Fushimi-ku, Kyoto, 612-8364, Japan, Telephone: +81756211111; Fax; +81756231117; E-mail: svbsystem@yahoo.co.jp

Key words: lag screw, offset, intertrochanteric fracture, computed tomography

Received: December 03, 2019; Accepted: December 17, 2019; Published: December 20, 2019 


\section{Patients and methods}

We evaluated 100 patients with intertrochanteric fractures treated with IHN at two participating hospitals from 2002-2018. These cases were randomly selected from 237 cases of femoral intertrochanteric fractures. Surgeries were performed in the supine position on a traction table with image intensifier guidance. Reduction was performed in a standard manner by traction and adduction of the leg, and the reduction was confirmed by anteroposterior and lateral images. A guidewire was then introduced from the tip of the greater trochanter, followed by inserting the femoral nail, after reaming. The position of the tip of the lag screw was aimed at the center or the inferior half of the femoral head on the anteroposterior view and the center of the femoral head on the lateral view.

The femoral neck was divided into three areas along the axial plane of the CT images (A: Anterior, C: Central and P: Posterior), and the position of the lag screw relative to the femoral neck was categorized (Figure 1). The minimum distance between the central axis of the femoral neck and the center of the nail was measured, and posterior positioning of the nail was considered positive positioning. The reduction status of the fracture and the position of the lag screw were assessed by postoperative CT. "Anatomical reduction" in this study was defined as no displacement $( \pm 1 \mathrm{~mm})$ in the fractured fragments between proximal bone including femoral head and distal bone including femoral shaft excluding trochanter major and minor; "posterior displacement" of the fragments was defined as a posteriorly displaced proximal fragment; and "anterior displacement" was defined as an anteriorly displaced proximal fragment. The maximal gap length between the anterior walls of the proximal and distal fragments was also measured (Figure 1).

The lag screw deviation from at the level of center of the nail to the tip of the lag screw was categorized. When both the point at the center of the nail and the tip of the screw were in the same area, the screw direction was defined as "no deviation". The lag screw was considered deviated when the point of the screw at the center of the nail and tip of the screw were in different areas, and was categorized as AP (from anterior to posterior) or PA (from posterior to anterior) according to the direction of the lag screw. The angle between the screw and the femoral neck axis was also measured, with anterior direction considered positive deviation.

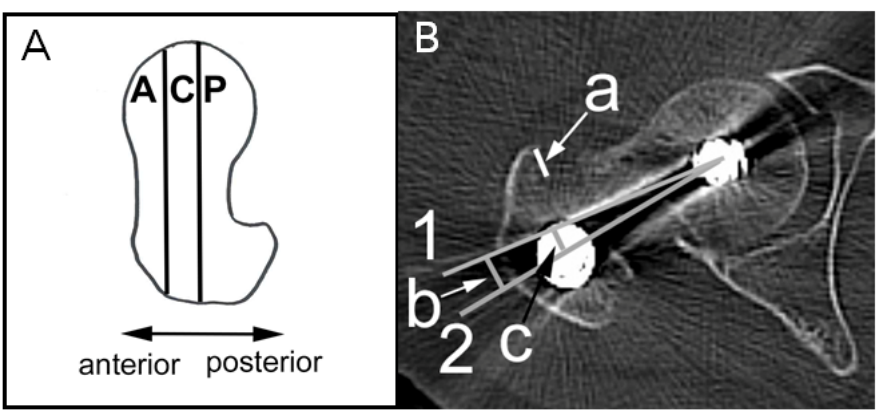

Figure 1. Reduction status of the fracture and position of the lag screw were assessed by axial plane CT. (A) The femoral neck was divided into three areas on axial plane CT images (zone A: Anterior, zone C: Central, and zone P: Posterior), and the position of the lag screw relative to the femoral neck were categorized. We measured the angle between the screw and the femoral neck axis and defined anterior deviation as positive. (B) Displacement of the fragment was quantified by measuring the maximal gap length between the anterior walls of the proximal and distal fragments (a). We assessed the position of the lag screw relative to the femoral neck by measuring the angle (b) between the central axis of the femoral neck (1) and the lag screw (2). The minimum distance (c) between the central axis of the femoral neck (1) and the center of the nail (2) was measured. We defined posterior positioning of the screw as positive. CT, computed tomography

\section{Results}

We analyzed data for 100 patients with intertrochanteric fracture treated with IHN (23 men and 77 women). The mean age of the patients at the time of surgery was mean age $83.9 \pm 8.9$ years (61 to 96 ). Patients' mean body height was $149.1 \pm 9.2 \mathrm{~cm}$ (132 to 172$)$, and the mean follow-up period following surgery was $33.4 \pm 29.9$ months (1.2 to 165.5). Fracture types according to the AO Foundation and Orthopedic Trauma Association (AO/OTA) classification was 31-A1 in 20 patients and $31-\mathrm{A} 2$ in 80 patients. Multidetector CT images with 1-mm slice thickness were obtained $7.9 \pm 16.5 \mathrm{~m}$ (0.1 to 110.8$)$ postoperatively.

The position of the lag screw at the nail level was in area $\mathrm{A}$ in 8 patients, area $\mathrm{C}$ in 15 patients, and area $\mathrm{P}$ in 77 patients. The distance between the lag screw on the center of the nail and the femoral neck axis was $4.6 \pm 2.8 \mathrm{~mm}(-4.0$ to 12.6$)$.

The proximal fragment was posteriorly displaced (posterior displacement) in 82 patients, anteriorly displaced (anterior displacement) in 1 patient, and nondisplaced in 17 patients (17.0\%). The displacement distance in the posterior displacement cases was $4.6 \pm 2.8 \mathrm{~mm}$ ( -3.8 to 12.0$)$.

Regarding deviation of the lag screw direction, 39 patients were non-deviated: $\mathrm{A}$ in 2 patients, $\mathrm{C}$ in 12 patients, and $\mathrm{P}$ in 25 patients. In most of the non-deviated patients, the proximal fragment was posteriorly displaced (33/39 patients) (Table 1$)$. Deviation of the screw direction was observed in 61 patients: AP in 7 patients and PA in 54 patients, indicating that deviation from posterior to anterior was dominant (Table 1). The deviation angle of the lag screw was $11.6 \pm 10.9^{\circ}$ (7.2 to 37.5).

\section{Representative patient}

A 93-year-old woman was diagnosed with intertrochanteric fracture (AO/OTA 31-A2, unstable type) and underwent IHN (Figure 2). CT demonstrated that the nail was inserted posterior to area $\mathrm{C}$, and that the direction of the screw was PA (deviation angle, $16.7^{\circ}$ ). Full weight-bearing was allowed 3 weeks postoperatively. Cut-out was confirmed by radiography 25 days postoperatively, and she underwent hip hemiarthroplasty.

\section{Discussion}

In this study, we analyzed the frequency of anterior/posterior displacement of intertrochanteric fractures and deviation of the lag screw after IHN confirmed using CT evaluation. Regarding reduction of the fracture fragments, $82 \%$ of the fragments were posteriorly displaced even though we confirmed fracture reduction using an image intensifier before inserting the nails. When the screw was inserted parallel with the femoral neck in the axial plane (zone A, zone $\mathrm{C}$, or zone $\mathrm{P}), 84.6 \%(34 / 39)$ of patients had displaced fragments, and most (33 patients) were posteriorly displaced. Conversely, in 17 nondisplaced cases, PA deviation of the screw direction was observed in 12 patients (70.6\%) (Table 1). These results suggest a disadvantage of IHN for internal fixation of intertrochanteric fractures because achieving both simultaneous anatomical reduction (at least no displacement in anterior or posterior direction between proximal bone, femoral head side, and distal bone, femoral shaft side) and proper positioning of the lag screw was rare.

We speculate that this finding was caused by anterior offset of the femoral neck relative to the femoral shaft. Toogood et al. analyzed 200 disarticulated adult skeletons, and found that the femoral head, and therefore, the femoral neck, tended to be deviated anteriorly 
Yamada I (2019) Analysis of reduction status and lag screw direction in intertrochanteric fractures treated with intramedullary nailing: Possible influence of anterior femoral head-neck offset

Table 1. The relationship between the reduction status and the lag screw direction

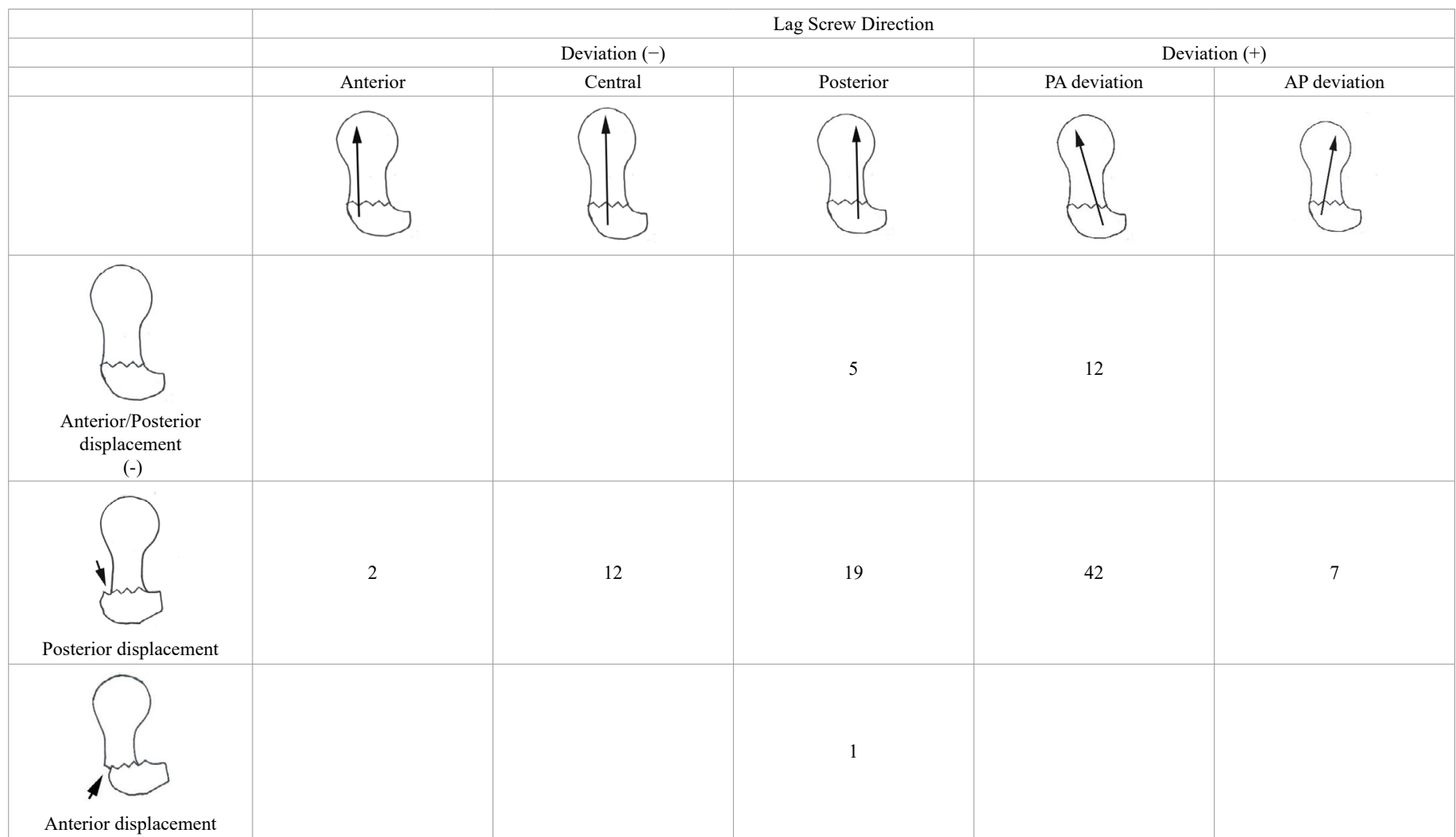

Numbers indicate the number of patients. PA: posterior-anterior; AP: anterior-posterior
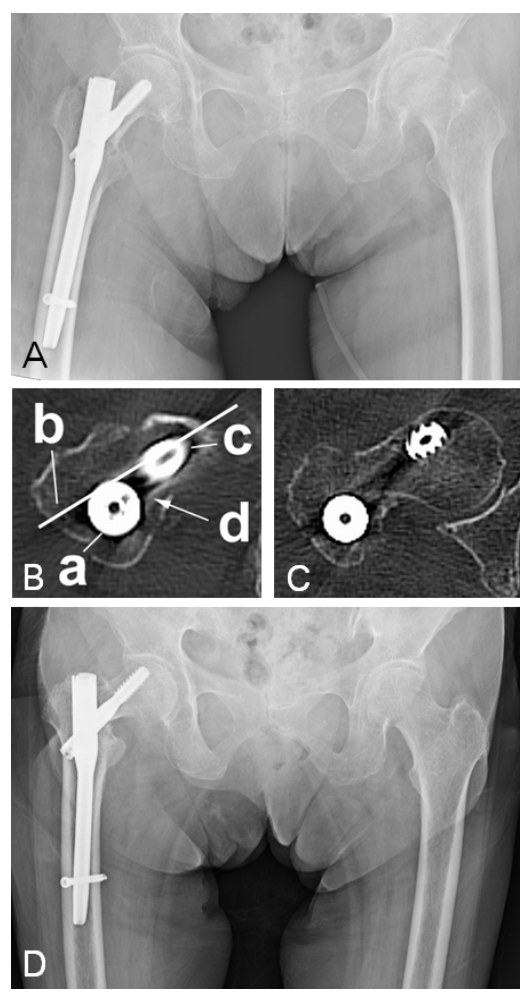

Figure 2. Case presentation: (A) A 93-year-old women with a right intertrochanteric fracture (AO/OTA 31-A2) underwent IHN, for fracture repair. (B \& C) Axial plane CT images showing that the lag screw at the nail level (a) is positioned at area P. Note that the lag screw touches the posterior wall of the femoral neck (arrow). The surgeon instinctively choses a route with less resistance, preventing accidents. As a result, the lag screw was inserted from posterior to anterior to prevent the wall damage. (D) Cut-out occurred 25 days postoperatively.

AO/OTA, AO Foundation and Orthopedic Trauma Association; IHN, intramedullary hip nail; CT, computed tomography 


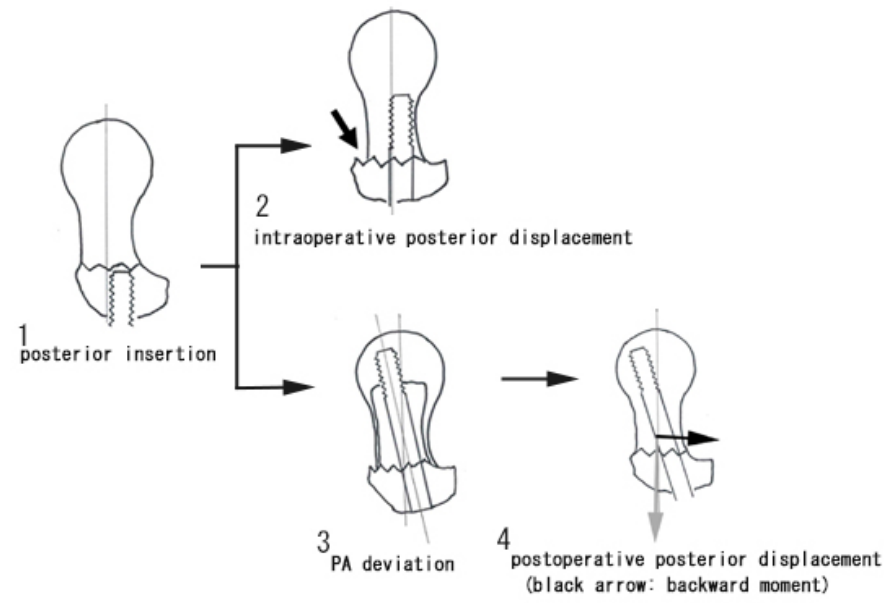

Figure 3. A possible mechanism explaining how anterior offset of the femoral neck affects the reduction status and the direction of the lag screw. Because of the anterior offset, the lag screw at the nail level is placed posteriorly relative to the femoral neck axis (1). When the lag screw is introduced parallel with the femoral neck, the screw may hit the posterior wall of the femoral neck. If the lag screw is inserted deeply, intraoperative posterior displacement (arrow) because the distal part of the bone is spreading, or destruction of the posterior wall may occur (2). To avoid this, lag screws are frequently inserted from posterior to anterior (3). This third figure is visualized results of this study. Backward moment (black arrow) by PA deviation may become the cause of postoperative posterior displacement (4)

[21]. We also confirmed anterior offset of the femoral neck relative to the femoral shaft, in our study, because lag screw at the level of the intramedullary nail, namely the nail itself, was positioned at area $\mathrm{P}$ in $77 \%$ of patients. Because of this anterior offset of the femoral neck, when the lag screw is introduced parallel with the femoral neck, the screw may hit the posterior wall of the femoral neck, which leads to posterior displacement of the fragment or even to destruction of the posterior wall (Figure 3). We observed defects in the posterior wall in approximately $10 \%$ of the patients in this study. To avoid this, lag screws were frequently inserted in the PA direction (Table 1); however, deviation of the lag screw in this direction may cause reduced mechanical strength by shearing forces, and result in malalignment of the fracture and/or postoperative posterior displacement by backward moment (Figure 3) [20,22-24].

In conclusion, we found it difficult to achieve simultaneous anatomical reduction, at least between femoral head and femoral shaft in anterior/posterior direction, and proper positioning: zone $\mathrm{C}$ of the lag screw in IHN surgeries. We suspect this was because of anterior offset of the femoral neck relative to the femoral shaft. Therefore, new intramedullary nail designs such as nails with anterior offset are needed to achieve better CT results with IHN for intertrochanteric fracture repair.

\section{References}

1. Hernlund E, Svedbom A, Ivergard M, Compston J, Cooper C, et al. (2013) Osteoporosis in the european union: Medical management, epidemiology and economic burden. A report prepared in collaboration with the international osteoporosis foundation (IOF) and the european federation of pharmaceutical industry associations (EFPIA). Arch Osteoporos 8: 130-136.

2. Svedbom A, Hernlund E, Ivergard M, Compston J, Cooper C, et al. (2013) Osteoporosis in the european union: A compendium of country-specific reports. Arch Osteoporos 8: 130-137.

3. Compston JE, McClung MR, Leslie WD (2019) Osteoporosis. Lancet 393: 364-376. [Crossref]

4. Cummings SR, Melton LJ (2002) Epidemiology and outcomes of osteoporotic fractures. Lancet 359: 1761-1767. [Crossref]
5. Yonezawa T, Yamazaki K, Atsumi T, Obara S (2009) Influence of the timing of surgery on mortality and activity of hip fracture in elderly patients. J Orthop Sci 14: 566-573. [Crossref]

6. Davis TR, Sher JL, Horsman A, Simpson M, Porter BB, et al. (1990) Intertrochanteric femoral fractures. Mechanical failure after internal fixation. J Bone Joint Surg Br 72 26-31. [Crossref]

7. Platzer P, Thalhammer G, Wozasek GE, Vécsei V (2008) Femoral shortening after surgical treatment of trochanteric fractures in nongeriatric patients. J Trauma 64: 982989. [Crossref]

8. Sadowski C, Lübbeke A, Saudan M, Riand N, Stern R, et al. (2002) Treatment of reverse oblique and transverse intertrochanteric fractures with use of an intramedullary nail or a 95 degrees screw-plate: a prospective, randomized study. $J$ Bone Joint Surg Am 84: 372-381. [Crossref]

9. Utrilla AL, Reig JS, Munoz FM, Tufanisco CB (2005) Trochanteric gamma nail and compression hip screw for trochanteric fractures: a randomized, prospective, comparative study in 210 elderly patients with a new design of the gamma nail. $J$ Orthop Trauma 19: 229-233.

10. Mavrogenis AF, Panagopoulos GN, Megaloikonomos PD, Igoumenou VG Galanopoulos I, et al. (2016) Complications after hip nailing for fractures. Orthopedics 39: e108-116. [Crossref]

11. Parker MJ, Handoll HH (2010) Gamma and other cephalocondylic intramedullary nails versus extramedullary implants for extracapsular hip fractures in adults. Cochrane Database Syst Rev 9: CD000093.

12. Kim TY, Lee YB, Chang JD, Lee SS, Yoo JH, et al. (2015) Torsional malalignment, how much significant in the trochanteric fractures? Injury 46: 2196-2200. [Crossref]

13. Ramanoudjame M, Guillon P, Dauzac C, Meunier C, Carcopino JM (2010) CT evaluation of torsional malalignment after intertrochanteric fracture fixation. Orthop Traumatol Surg Res 96: 844-848. [Crossref]

14. Jaarsma RL, Pakvis DF, Verdonschot N, Biert J, van Kampen A (2004) Rotational malalignment after intramedullary nailing of femoral fractures. J Orthop Trauma 18: 403-409. [Crossref]

15. Bojan AJ, Beimel C, Taglang G, Collin D, Ekholm C, et al. (2013) Critical factors in cut-out complication after gamma nail treatment of proximal femoral fractures. $B M C$ Musculoskelet Disord 14: 1-10.

16. Baumgaertner MR, Curtin SL, Lindskog DM, Keggi JM (1995) The value of the tipapex distance in predicting failure of fixation of peritrochanteric fractures of the hip. $J$ Bone Joint Surg Am 77: 1058-1064. [Crossref]

17. Larsson S, Friberg S, Hansson LI (1990) Trochanteric fractures influence of reduction and implant position on impaction and complications. Clin Orthop Relat Res 259: 130139.

18. Kyle RF, Cabanela ME, Russell TA, Swiontkowski MF, Winquist RA, et al. (1995) Fractures of the proximal part of the femur. Instr Course Lect 44: 227-253. [Crossref]

19. Poyanli OS, Soylemez S, Ozkut AT, Uygur E, Kemah B, et al. (2015) Precise placement of lag screws in operative treatment of trochanteric femoral fractures with a new guide system. Injury 46: 2190-2195. [Crossref]

20. Wu CC, Shih CH, Lee MY, Tai CL (1996) Biomechanical analysis of location of lag screw of a dynamic hip screw in treatment of unstable intertrochanteric fracture. $J$ Trauma 41: 699-702. [Crossref]

21. Toogood PA, Skalak A, Cooperman DR (2009) Proximal femoral anatomy in the normal human population. Clin Orthop Relat Res 467: 876-885. [Crossref]

22. Aro HT, Wahner HT, Chao EY (1991) Healing patterns of transverse and oblique osteotomies in the canine tibia under external fixation. J Orthop Trauma 5: 351-364. [Crossref]

23. Augat P, Burger J, Schorlemmer S, Henke T, Peraus M, et al. (2003) Shear movement at the fracture site delays healing in a diaphyseal fracture model. J Orthop Res 21 : 1011-1017.

24. Yamagishi M, Yoshimura Y (1955) The biomechanics of fracture healing. J Bone Joint Surg Am 37-37: 1035-68. [Crossref]

Copyright: (C2019 Yamada I. This is an open-access article distributed under the terms of the Creative Commons Attribution License, which permits unrestricted use, distribution, and reproduction in any medium, provided the original author and source are credited. 